Interfaces and Free Boundaries 12 (2010), 279-291

DOI $10.4171 / \mathrm{IFB} / 235$

\title{
Boundary regularity for a parabolic obstacle type problem
}

\author{
J. ANDERSSON \\ Mathematics Institute, University of Warwick, Coventry CV4 7AL, UK \\ E-mail: j.e.andersson@warwick.ac.uk
}

[Received 6 November 2008 and in revised form 26 April 2010]

We study the regularity of the free boundary, near contact points with the fixed boundary, for a parabolic free boundary problem

$$
\begin{array}{ll}
\Delta u-\partial u / \partial t=\chi\{u \neq 0\} & \text { in } Q_{r}^{+}=\left\{(x, t) \in B_{r} \times\left(-r^{2}, 0\right) ; x_{1}>0\right\}, \\
u=f(x, t) & \text { on }\left\{x_{1}=0\right\} \cap Q_{r} .
\end{array}
$$

We will show that under certain regularity assumptions on the boundary data $f$ the free boundary is a $C^{1}$ manifold up to the fixed boundary. We also show that the $C^{1}$ modulus of continuity is uniform for a certain, and specified, subclass of solutions.

\section{Introduction}

In this paper we will investigate the free boundary (see below for definitions) for a parabolic obstacle-like problem near contact points with the fixed boundary. Mathematically the problem can be formulated in the following way:

$$
\begin{cases}\Delta u-\partial_{t} u=\chi_{\Omega_{u}} & \text { in } Q_{1}^{+} \text {where } \Omega_{u}=Q_{r}^{+} \backslash\{u=|\nabla u|=0\}, \\ \left.u\right|_{\Pi}=f\left(x_{2}, \ldots, x_{n}, t\right) & \text { on } \Pi=\left\{x_{1}=0\right\} .\end{cases}
$$

All the relevant notation will be defined at the end of the introduction.

Before we state our main results we will briefly describe the mathematical and applicational context of our problem.

Applications: Under the assumption that $u, u_{t} \geqslant 0$ this is the well known Stefan problem describing the melting process of ice. For further details on the Stefan problem see [F].

Mathematical background: The regularity of free boundaries has been extensively studied over the last thirty years and the literature on the Stefan problem is vast. This problem however (that is, the Stefan problem without sign restriction) was, to the author's knowledge, first studied by L. A. Caffarelli, A. Petrosyan and H. Shahgholian in [CPS]. The authors of [CPS] showed that a solution is $C^{1,1}$ in space and Lipschitz in time, and that the free boundary is locally analytic under an assumption on the density of $\{u=0\}$ backward in time (see Definition 4 and Theorem 4).

The regularity of the free boundary near contact points with the fixed boundary was investigated by D. E. Apushkinskaya, N. N. Uraltseva and H. Shahgholian in [ASU1]. The authors of [ASU1] consider the free boundary close to a fixed boundary with zero Dirichlet condition. They also assume that $u \geqslant 0$. Under these conditions they prove that the free boundary is uniformly $C^{1, \alpha}$ away from the fixed boundary and Lipschitz as a graph over the fixed boundary near a contact point.

Recently D. E. Apushkinskaya, N. Matevosyan and N. N. Uraltseva AMU] extended the results of [ASU1] to solutions without sign restriction. 
Our main objective in this paper is to extend the results of [ASU1] to non-vanishing boundary data. In a sense this is a twin paper to $[\overline{\mathrm{A}}]$ where the same problem is investigated for a corresponding elliptic problem.

Plan of the paper: In the next section, after this short introduction, we will state our main results in Theorems 1-3. Before we are able to prove these theorems we must introduce some technical tools, the so-called monotonicity formulas. The monotonicity formulas are well known in this context, but we include them for completeness in section 3.

With these tools at hand we will prove the main theorems in Sections 4-6.

\section{Notation.}

- $\mathbb{R}^{n+1}$ will denote $n+1$-dimensional real space with coordinates $\left(x_{1}, \ldots, x_{n}, t\right)$.

- $B_{r}\left(x^{0}\right)$ will denote the $n$-dimensional ball in the $x$ variables with radius $r$ and center $x^{0}$.

- $Q_{r}\left(x^{0}, t^{0}\right)$ will denote $B_{r}\left(x^{0}\right) \times\left(-r^{2}, 0\right)$, the parabolic cylinder.

- $\mathbb{R}_{+}^{n}, B_{r}^{+}\left(x^{0}\right)$ and $Q_{r}^{+}\left(x^{0}, t^{0}\right)$ will denote the corresponding sets intersected with $\left\{x_{1}>0\right\}$.

- $\Pi$ will be the plane $\left\{x_{1}=0\right\}$.

- $\mathcal{H}^{n}(\Omega)$ is the $n$-dimensional Hausdorff measure of $\Omega$.

- $|\Omega|$ will denote the Lebesgue measure of $\Omega,|x|$ will also denote the euclidean norm of the vector $x \in \mathbb{R}^{n}$. With a slight abuse of notation we will use $|(x, t)|=\sqrt{|x|^{2}+|t|}$, the parabolic distance.

- $\operatorname{dist}\left(\left(x^{0}, t^{0}\right), \Omega\right)$ will denote the euclidean distance from $x^{0}$ to the set $\Omega \cap\left\{t=t^{0}\right\}$, the $t^{0}$-section of $\Omega$.

- $\operatorname{pardist}\left(\left(x^{0}, t^{0}\right), \Omega\right)$ will denote the parabolic distance between $\left(x^{0}, t^{0}\right)$ and $\Omega$, that is, $\inf _{(x, t) \in \Omega} \sqrt{\left|x-x^{0}\right|^{2}+\left|t-t^{0}\right|}$.

- $\chi_{\Omega}$ will denote the characteristic function of the set $\Omega$.

- $u^{ \pm}$will denote the positive and negative parts of the function $u$, that is, $u^{ \pm}=\max ( \pm u, 0)$.

- $\left.f\right|_{\Omega}$ will denote the restriction of $f$ to the set $\Omega$.

- $\partial_{i}$ for $i=1, \ldots, n$ and $\partial_{t}$ will denote $\partial / \partial x_{i}$ and $\partial / \partial t$ respectively.

- $u_{i}$ and $u_{t}$ will be used for $\partial_{i} u$ and $\partial_{t} u$ respectively.

- $\Delta$ is the spatial laplacian, $\Delta=\sum_{i=1}^{n} \partial_{i}^{2}$.

- $\nabla u$ is the spatial gradient of $u, \nabla u=\left(\partial_{1} u, \ldots, \partial_{n} u\right)$.

- $\Lambda_{u}$ is the set where $u=|\nabla u|=0$. We will also use the notation $\Lambda_{u}\left(-r^{2}\right)$ for $\Lambda_{u} \cap\left\{t=-r^{2}\right\}$.

- $\Omega_{u}$ is the complement of $\Lambda_{u}$ with respect to the domain of $u$.

- $\Gamma_{u}$, the free boundary of $u$, is the intersection of the closures of $\Lambda_{u}$ and $\Omega_{u}$.

- $C^{1+\alpha, \beta}(\Omega)$ is the parabolic Hölder space of functions $C^{1+\alpha}$ in the spatial variables and $C^{\beta}$ in the time variable.

- $W_{a}(r, u)$ is defined in Lemma 1 .

- $P_{r}(M, f)$ is defined in Definition 1 .

- $\operatorname{cap}(A)$ will denote the parabolic capacity of the set $A$ (see [Wa]).

- The $\operatorname{limit} \lim _{r \rightarrow 0} u\left(r x, r^{2} t\right) / r^{2}$, at times normalized differently (e.g. $\lim _{r \rightarrow 0} u\left(r x, r^{2} t\right) \times$ $\left.\left(\sup _{Q_{r}}|u|\right)^{-1}\right)$, through some subsequence will be called the blow-up of $u$.

\section{Main results}

Before we state our main results we need some definitions to simplify our statements. First, we will always assume that $f \in C^{1,1}(\Pi)$. 
DEFINITION $1 P_{r}(M, f)$ is the set of all functions solving equation (1) in the sense of distributions and whose $L^{\infty}$-norm is bounded by $M$.

By $P_{\infty}(f)$ we will mean the set of quadratically bounded functions solving equation (1) in the sense of distributions in the entire space $x_{1}>0, t<0$. Here "quadratically bounded" means $\sup _{Q_{r}^{+}}|u|<C r^{2}$, where $C$ may depend on $u$ but not on $r$.

THEOREM 1 Let $u \in P_{1}(M, f)$ and

$$
\liminf _{r \rightarrow 0} \frac{\operatorname{cap}\left(\Lambda_{u} \cap Q_{r}\right)}{\operatorname{cap}\left(Q_{r}\right)}>\epsilon>0 \text { and } \sup _{Q_{r} \cap \Pi}|f| \leqslant C_{1} r^{2} .
$$

Then

$$
\sup _{Q_{r}^{+}}|u| \leqslant C_{2} M r^{2} .
$$

The assumption on the density of the capacity is necessary in Theorem 1. In general we do not get quadratic bounds for solutions to parabolic problems with $C^{1,1}$ data. The free boundary introduces some extra freedom in the problem that improves the regularity of the solution.

Next we classify the solutions with homogeneous boundary data.

THEOREM 2 Let $u \in P_{\infty}(f)$ be a homogeneous function. Then if $f=\left.\frac{1}{2}(e \cdot x)_{+}^{2}\right|_{\Pi}$ for a unit vector $e=\left(e_{1}, \ldots, e_{n}\right) \in \mathbb{R}^{n}$, then $u=\frac{1}{2}(e \cdot x)_{+}^{2}$ or $u=\frac{1}{2}(\hat{e} \cdot x)_{+}^{2}$ in $Q_{\infty}^{+}$. Here $\hat{e}=\left(-e_{1}, e_{2}, \ldots, e_{n}\right)$ is the reflection of $e$ in $\Pi$.

REMARK An interesting question is whether the same result is true without the homogeneity assumption. This is not the case; for a proof see [AS.

Because of the importance of the two half polynomial solutions introduced in the above theorem we will make the following definition.

DEFINITION 2 Let $f$ be a given function on $\Pi$ satisfying

$$
\lim _{r \rightarrow 0} \frac{f(r x)}{r^{2}}=\left.(e \cdot x)_{+}^{2}\right|_{\Pi}
$$

for some $e$ with $|e|=1$. By $U_{1}^{f}$ we mean $\frac{1}{2}\left(\left(-\left|e_{1}\right|, e_{2}, \ldots, e_{n}\right) \cdot x\right)_{+}^{2}$ and by $U_{2}^{f}$ we mean $\frac{1}{2}\left(\left(\left|e_{1}\right|, e_{2}, \ldots, e_{n}\right) \cdot x\right)_{+}^{2}$.

We will often write $U_{1}$ and $U_{2}$ when the $f$-dependence is given by context.

For the use of the monotonicity formula in Lemma 1 we will also need another assumption on $f$.

Condition 1 Let $f_{r}(x, t)=f\left(r x, r^{2} t\right) / r^{2}$. Then $f$ satisfies Condition 1 if $\lim _{r \rightarrow 0} f_{r}=\lambda\left(x_{2}\right)_{+}^{2}$ for some $0<\lambda \leqslant 1 / 2$ and

$$
\left|\int_{-4}^{-1} \int_{\Pi \cap B_{a / r}}\right| f_{r}^{\prime}|G(x,-t) \mathrm{d} x \mathrm{~d} t| \leqslant C_{h}(r)
$$

for some Dini modulus of continuity $C_{h}: \int_{0}^{1 / 2} r^{-1} C_{h}(r) \mathrm{d} r<\infty$.

In particular if Condition 1 is satisfied for some $C_{h}$ then the blow-up is unique (see Lemma 3 ).

The final main result in this paper regards the local behavior of the free boundary near a contact point with the fixed boundary. As in $[\mathrm{A}]$ this result depends on the blow-up of $u$ at the contact point. We will need the following definition. 
DEFINITION $3 \hat{P}_{r}(M, f)$ will denote the subset of $P_{r}(M, f)$ of functions whose blow-up is unique at the origin and equals $U_{2}$.

Here we tacitly assume that the blow-up of $f$ is a half polynomial.

THEOREM 3 Let $u \in \hat{P}_{1}(M, f)$, let $\Gamma_{u}$ satisfy the capacity condition

$$
\liminf _{r \rightarrow 0} \frac{\operatorname{cap}\left(\Lambda_{u} \cap Q_{r}\right)}{\operatorname{cap}\left(Q_{r}\right)}>\epsilon>0
$$

for some $\epsilon>0$, and assume furthermore that $f$ satisfies Condition 1 Then for each $\gamma>0$ :

1. There exists a modulus of continuity $\sigma_{\gamma}$ and a universal constant $r_{0}=r_{0}\left(n, M, \gamma, C_{h}, \epsilon\right)$ such that

$$
\Gamma_{u} \cap C_{r_{0}}^{\gamma} \subset\left\{x ; \operatorname{pardist}\left((x, t), \partial \Omega_{U_{2}}\right) \leqslant \sigma_{\gamma}(|(x, t)|)|(x, t)|\right\},
$$

where $C_{r_{0}}^{\gamma}=Q_{r_{0}}^{+} \cap\left\{x_{1}>\gamma|(x, t)|\right\}$ is a parabolic nontangential cone.

2. $\Gamma_{u}$ is a $C^{1}$ manifold with uniform modulus of continuity (depending on $n, \gamma, \epsilon$ and $C_{h}$ from Condition 10 in $C_{r_{0}}^{\gamma}$.

REMARK The same is not true if the blow-up of $u$ equals $U_{1}: \Gamma_{u}$ will be a $C^{1}$ manifold, but it is not true that $\Gamma_{u}$ is uniformly $C^{1}$. The proof of this is somewhat simpler and very similar to the proof of Theorem 3 See $[\mathrm{A}]$ for details in the time independent case.

REMARK The theorem gives a pointwise regularity condition. It is easy, under some assumptions on $f$, to extend the theorem to a regularity result in $Q_{\delta}^{+}(0)$. In particular if $\partial \operatorname{spt}(f) \in C^{1, \alpha}\left(Q_{\delta}^{\prime}(0)\right)$ and $e=e\left(x^{\prime}, t\right)$ (from Definition 2) is a $C^{\alpha}$ function living on $\partial \operatorname{spt}(f)$ and the conditions in Theorem 3 are satisfied pointwise on $\partial \operatorname{spt}(\mathrm{f})$ then $\Gamma \in C^{1, \alpha}\left(\overline{Q_{\delta / 2}^{+}(0)}\right)$.

\section{Technical tools and known results}

An essential role in the theory of free boundaries is played by the so-called monotonicity formulas. We will use a monotonicity formula due to G. S. Weiss.

LEMMA 1 Let $u$ be a solution to (1). Then

$$
W_{a}(r, u)=\frac{1}{r^{4}} \int_{-4 r^{2}}^{-r^{2}} \int_{B_{a}^{+}}\left(\frac{1}{2}|\nabla u|^{2}+u+\frac{u^{2}}{2 t}\right) G(x, t) \mathrm{d} x \mathrm{~d} t
$$

satisfies

$$
\begin{aligned}
& \frac{\mathrm{d} W_{a}(r, u)}{\mathrm{d} r} \\
& =\frac{1}{2 r} \int_{-4}^{-1} \int_{B_{a / r}^{+}} \frac{\left|u_{r}^{\prime}\right|^{2}}{-t} G(x,-t) \mathrm{d} x \mathrm{~d} t+J_{a}(r, u)-\int_{-4}^{-1} \int_{\Pi \cap B_{a / r}} f_{r}^{\prime}\left(\frac{\partial u_{r}}{\partial x_{1}}\right) G(x,-t) \mathrm{d} x \mathrm{~d} t
\end{aligned}
$$


for $0<r \leqslant a \leqslant 1$. Here

$$
\begin{aligned}
u_{r}(x, t)= & \frac{u\left(r x, r^{2} t\right)}{r^{2}}, \\
u_{r}^{\prime}(x, t)= & x \cdot \nabla u_{r}(x, t)+2 t \partial_{t} u_{r}(x, t)-2 u_{r}(x, t), \\
G(x, t)= & \frac{\exp \left(-|x|^{2} / 4 t\right)}{(4 \pi t)^{n / 2}} \text { for } t>0 \text { and } \quad G(x, t)=0 \quad \text { for } t \leqslant 0, \\
J_{a}(r, u)= & \int_{-4}^{-1} \int_{\left(\partial B_{r / a}\right)^{+}} \frac{u_{r}^{\prime}}{r}\left(\eta \cdot \nabla u_{r}\right) G(x,-t) \mathrm{d} x \mathrm{~d} t \\
& -\frac{a}{2 r^{2}} \int_{-4}^{-1} \int_{\left(\partial B_{a / r}\right)^{+}}\left(\left|\nabla u_{r}\right|^{2}+2 u_{r}+\frac{\left(u_{r}\right)^{2}}{t}\right) G(x,-t) \mathrm{d} x \mathrm{~d} t,
\end{aligned}
$$

and $\eta$ is the outward unit normal of $\partial B_{a / r}^{+}$.

Proof. The proof follows the same lines as in [ASU]. By a change of variables we see that

$$
W_{a}(r, u)=W_{a / r}\left(1, u_{r}\right)
$$

where $u_{r}=u\left(r x, r^{2} t\right) / r^{2}$. From this we get

$$
\begin{aligned}
\frac{\mathrm{d}}{\mathrm{d} r} W_{a / r}= & \int_{-4}^{-1} \int_{B_{a / r}^{+}}\left(\nabla u_{r}^{\prime} \cdot \nabla u_{r}+u_{r}^{\prime}+\frac{u_{r} u_{r}^{\prime}}{t}\right) G(x,-t) \mathrm{d} x \mathrm{~d} t \\
& -\frac{a}{r^{2}} \int_{-4}^{-1} \int_{\partial B_{a / r} \cap \mathbb{R}_{+}^{n}}\left(\frac{1}{2}\left|\nabla u_{r}\right|^{2}+u_{r}+\frac{u_{r}^{2}}{2 t}\right) G(x,-t) \mathrm{d} x \mathrm{~d} t=I_{1}+I_{2} .
\end{aligned}
$$

Integrating $I_{1}$ by parts leads to

$$
\begin{aligned}
I_{1}= & \int_{-4}^{-1} \int_{B_{a / r}^{+}} u_{r}^{\prime}\left(-\Delta u_{r}-\frac{x_{i}}{2 t} \partial_{i} u_{r}+1+\frac{u_{r}}{t}\right) G(x,-t) \mathrm{d} x \mathrm{~d} t \\
& +\int_{-4}^{-1} \int_{\partial B_{a / r}^{+} \cap \mathbb{R}_{+}^{n}} u_{r}^{\prime} \frac{\partial u_{r}}{\partial \eta} G(x,-t) \mathrm{d} x \mathrm{~d} r-\int_{-4}^{-1} \int_{\Pi \cap B_{a / r}} f_{r}^{\prime}\left(\frac{\partial u_{r}}{\partial x_{1}}\right) G(x,-t) \mathrm{d} x \mathrm{~d} t,
\end{aligned}
$$

where $\eta$ is the outward unit normal. Here we are using that $u=f$ on the boundary $\left\{x_{1}=0\right\}$.

Using the definition of $u_{r}^{\prime}$ together with the equality

$$
\frac{\mathrm{d} u}{\mathrm{~d} r}=\frac{u_{r}^{\prime}}{r}
$$

leads to

$$
-\Delta u_{r}-\frac{x_{i}}{2 t} \partial_{i} u_{r}+1+\frac{u_{r}}{t}=-\Delta u_{r}+\partial_{t} u_{r}+1-\frac{u_{r}^{\prime}}{2 t} .
$$

Using this in equation (3) gives the desired result. 
COROLlary 1 Let $u$ be a solution to 11 and assume that $\sup _{Q_{s}^{+}}|u| \leqslant C s^{2}$ for all $s<1$ and some constant $C$ independent of $s$. Then, in the notation of Lemma 1 .

1. We have

$$
\left|J_{a}(r, u)\right| \leqslant \frac{C}{p(r / a)} e^{-\frac{a^{2}}{4 r^{2}}}
$$

where $p>0$ and goes to 0 as $r \rightarrow 0$ with polynomial speed.

2. If $f$ satisfies Condition 1 then the $\operatorname{limit} \lim _{r \rightarrow 0} W_{a}(r, u)$ exists.

Proof. Since $u$ is a solution and $\sup _{Q_{s}^{+}}|u| \leqslant C s^{2}$ it follows that $\left|\nabla u_{r}\right| \leqslant C a / r,\left|u_{r}^{\prime}\right| \leqslant C(a / r)^{2}$ and $\left|u_{r}\right| \leqslant C(a / r)^{2}$ on $\partial B_{a / r} \times(-1,-4)$. Substituting these estimates in the expression for $J_{a}(r, u)$ directly implies the first part of the corollary.

As for the second part we notice that by standard $C^{1, \alpha}$ estimates,

$$
\sup _{Q_{s} \cap\left\{x_{1}=0\right\}}\left|\frac{\partial u_{r}}{\partial x_{1}}\right| \leqslant \frac{C}{s} \sup _{Q_{2 s}^{+}}\left|u_{r}\right| \leqslant C s,
$$

where we have used the assumption that $\sup _{Q_{s}^{+}}|u| \leqslant C s^{2}$. It follows that

$$
\begin{aligned}
\left|\int_{-4}^{-1} \int_{\Pi \cap B_{a / r}} f_{r}^{\prime}\left(\frac{\partial u_{r}}{\partial x_{1}}\right) G(x,-t) \mathrm{d} x \mathrm{~d} t\right| & \leqslant C \int_{-4}^{-1} \int_{\Pi \cap B_{a / r}}\left|f_{r}^{\prime}\right|(1+|x|) G(x,-t) \mathrm{d} x \mathrm{~d} t \\
& \leqslant C \int_{-4}^{-1} \int_{\Pi \cap B_{a / r}}\left|f_{r}^{\prime}\right| \frac{a}{r} G(x,-t) \mathrm{d} x \mathrm{~d} t .
\end{aligned}
$$

From Lemma 1 it follows that for $0<s, t<1$ we have

$$
\begin{aligned}
W_{a}(t, u)- & W_{a}(s t, u)=\int_{s t}^{t} \frac{\mathrm{d} W_{a}(r, u)}{\mathrm{d} r} \mathrm{~d} r \\
\geqslant & \int_{s t}^{t}\left[\frac{1}{2 r} \int_{-4}^{-1} \int_{B_{a / r}^{+}} \frac{\left|u_{r}^{\prime}\right|^{2}}{-t} G(x,-t) \mathrm{d} x \mathrm{~d} t+J_{a}(r, u)\right. \\
& \left.-\int_{-4}^{-1} \int_{\Pi \cap B_{a / r}} f_{r}^{\prime}\left(\frac{\partial u_{r}}{\partial x_{1}}\right) G(x,-t) \mathrm{d} x \mathrm{~d} t\right] \mathrm{d} r \\
\geqslant & -C \mid \int_{s t}^{t} \frac{C}{p(r / a)} e^{-\frac{a^{2}}{4 r^{2}} \mathrm{~d} r\left|-C \int_{s t}^{t} \frac{a}{r}\right| \int_{-4}^{-1} \int_{\Pi \cap B_{a / r}}\left|f_{r}^{\prime}\right| G(x,-t) \mathrm{d} x \mathrm{~d} t \mid \mathrm{d} r=I_{1}+I_{2} .}
\end{aligned}
$$

The first claim in the corollary implies that $I_{1} \rightarrow 0$ as $t \rightarrow 0$ uniformly in $s$; and using that $f$ satisfies Condition 1 implies that $I_{2} \rightarrow 0$ as $t \rightarrow 0$ uniformly in $s$.

In particular it follows that

$$
W_{a}(t, u)-W_{a}(s t, u) \geqslant-\sigma(t),
$$

where $\sigma(t)$ is some modulus of continuity. This implies that

$$
\begin{aligned}
\limsup _{r \rightarrow 0} W_{a}(r, u) & =\lim _{t \rightarrow 0} \sup _{s \in(0,1)} W_{a}(s t, u)=\liminf _{t \rightarrow 0} \sup _{s \in(0,1)} W_{a}(s t, u) \\
& \leqslant \liminf _{t \rightarrow 0}\left(W_{a}(t, u)+\sigma(t)\right)=\liminf _{t \rightarrow 0} W_{a}(t, u) .
\end{aligned}
$$


It is also known that we have the following non-degeneracy of the solutions (for a proof see [CPS]).

LEMMA 2 Let $u$ be a solution to

$$
\Delta u-\frac{\partial u}{\partial t}=\chi\{u \neq 0\} \quad \text { in } Q_{2 r}
$$

and assume that $(0,0) \in \Gamma_{u}$. Then

$$
\sup _{Q_{r}}|u| \geqslant c r^{2}
$$

\section{Proof of Theorem 1}

The proof follows the lines of the proof of Lemma 3.1 in [ASU2]; we will sketch some details.

Denote

$$
M_{k}(u)=\sup _{Q_{2^{-k}}^{+}}|u| \quad \text { for } k \in \mathbb{N} .
$$

It is sufficient to show that there exists $C_{2}$ such that

$$
4^{k+1} M_{k+1}(u) \leqslant \max \left(4 M_{1}(u), \ldots, 4^{k} M_{k}(u), M C_{2}\right) \quad \forall k \in \mathbb{N} .
$$

Suppose, in order to get a contradiction, that this fails. That is, for every $j \in \mathbb{N}$ there exist $u_{j} \in$ $P_{1}^{+}(M)$ and $k_{j} \in \mathbb{N}$ such that

$$
4^{k_{j}+1} M_{k+1}\left(u_{j}\right) \geqslant \max \left(4 M_{1}\left(u_{j}\right), \ldots, 4^{k_{j}} M_{k}\left(u_{j}\right), j\right) .
$$

We now make the blow-up

$$
\tilde{u}_{j}=\frac{u_{j}\left(2^{-k_{j}} x, 2^{-2 k_{j}} t\right)}{M_{k_{j}+1}\left(u_{j}\right)} .
$$

By $C^{1, \alpha}$ regularity it follows that $k_{j} \rightarrow \infty$ as $j \rightarrow \infty$.

We will also need a growth estimate of $\tilde{u}_{j}$ :

$$
\sup _{Q_{2^{k}}^{+}}\left|\tilde{u}_{j}\right|=\sup _{Q_{2^{k}}^{+}} \frac{u_{j}\left(2^{-k_{j}} x, 2^{-2 k_{j}} t\right)}{M_{k_{j}+1}\left(u_{j}\right)}=\frac{M_{k_{j}-k}\left(u_{j}\right)}{M_{k_{j}+1}\left(u_{j}\right)} \leqslant 2^{2 k+2},
$$

that is, $\sup _{Q_{R}^{+}}\left|\tilde{u}_{j}\right| \leqslant C R^{2}$ for $R \geqslant 1$. We also get

$$
\left|\Delta \tilde{u}_{j}-\frac{\partial \tilde{u}_{j}}{\partial t}\right| \leqslant \frac{2^{-2 k_{j}}}{M_{k_{j}+1}}\left|\Delta u_{j}-\frac{\partial u_{j}}{\partial t}\right| \leqslant \frac{4}{j} \rightarrow 0 .
$$

It follows from the definition of $\tilde{u}_{j}$ that $\sup _{Q_{1 / 2}^{+}}\left|\tilde{u}_{j}\right|=\sup _{Q_{2^{-k_{j}-1}}^{+}} u_{j} / M_{k_{j}+1}=1$ so that

$$
\sup _{Q_{R}^{\prime}} \tilde{u}_{j}\left(0, x^{\prime}, t\right)=\sup _{Q_{R}^{\prime}} \frac{f\left(2^{-k_{j}} x^{\prime}, s^{-2 k_{j}} t\right)}{M_{k_{j}+1}} \leqslant \frac{4 C R^{2}}{j} \rightarrow 0 .
$$

So $\tilde{u}_{j} \rightarrow 0$ locally uniformly on $\Pi$. 
Standard regularity theory for parabolic equations implies that the $C^{1, \alpha}$ and $W^{2, p}$ norms of $\tilde{u}_{j}$ are uniformly locally bounded. This implies that a subsequence of $\tilde{u}_{j}$ will converge to, say, $u_{0}$ in $C_{\text {loc }}^{1, \alpha}$ and $W_{\text {loc }}^{2, p}$. Moreover $u_{0}$ will be a caloric, non-zero (since $\sup _{Q_{1 / 2}^{\prime}}\left|\tilde{u}_{j}\right|=1$ ), quadratically bounded (since $\tilde{u}_{j}$ is) function in $Q_{\infty}^{+}$with zero boundary values on $\Pi$.

Now we can use the Liuoville Theorem (Lemma 2.1 in [ASU1]) to deduce that $u_{0}$ is a quadratic polynomial in $x$ and linear in $t$. Using that $u_{0}=0$ on $\Pi$ we deduce that $u_{0}(x, t)=x_{1} \sum_{i=1}^{n} b_{i} x_{i}=$ $x_{1} \bar{b} \cdot x$ for some non-zero vector $\bar{b}=\left(b_{1}, \ldots, b_{n}\right)$. Since, by $\left.\sqrt{5}\right), \Delta u_{0}-\partial u_{0} / \partial t=0$ we can conclude that $b_{1}=0$.

By rotating the coordinate system we may assume that $\bar{b}=c e_{2}$ for some constant $c>0$. Since $\sup _{Q_{1}^{+}}|u|=1$ it follows that $c=2$ and therefore $u_{0}(x)=2 x_{1} x_{2}$. In particular cap $\left(\left\{x ; u_{0}(x)=\right.\right.$ $\left.\left.\left|\nabla u_{0}(x)\right|=0\right\} \cap Q_{1}\right)=0$. From that we will deduce a contradiction.

By $C^{1, \alpha}$ convergence we have $\limsup _{j \rightarrow \infty} \Lambda_{u_{j}} \subset \Lambda_{u_{0}}$, that is, if $x^{j} \in \Lambda_{u_{j}}$ and $x^{j} \rightarrow x^{0}$ then $x^{0} \in \Lambda_{u_{0}}$. Also by continuity $\Lambda_{u_{0}}$ is closed and thus (see [Wa]) cap $\left(\Lambda_{u_{0}} \cap Q_{1}\right)=\inf _{S} \operatorname{cap}(S)$ where the inf is taken over all open sets $S$ such that $\Lambda_{u_{0}} \cap Q_{1} \subset S$. We can thus find an open set $A$ such that $\Lambda_{u_{0}} \cap Q_{1} \subset A$ and $\operatorname{cap}(A)-\delta \leqslant \operatorname{cap}\left(\Lambda_{u_{0}} \cap Q_{1}\right)$. Also if $j$ is large enough then $\Lambda_{u_{j}} \cap Q_{1} \subset A$, so $\operatorname{cap}(A) \geqslant \operatorname{cap}\left(\Lambda_{u_{j}} \cap Q_{1}\right)$. Thus if $\delta$ is chosen small enough then $\operatorname{cap}\left(\Lambda_{u_{0}} \cap Q_{1}\right)>0$ and we get a contradiction.

\section{Proof of Theorem 2}

We will reduce the theorem to the time independent case and then refer the reader to the proof in $[\mathrm{A}]$. To this end let us denote

$$
v(x, t)=\left(\frac{\partial u(x, t)}{\partial t}\right)^{+},
$$

where we extend $v$ by zero to the lower half-space. The proof follows a number of simple steps:

1. $v$ is subcaloric in $\{t<0\}$,

2. $v \leqslant C$,

3. $v=0$.

Proof of Step 1. Since $\Delta u-u_{t}=1$ in $\Omega$ it follows that $v$ is caloric in $\Omega$. So we only need to show that $v$ is continuous across the free boundary; then it follows that $v$ is a non-negative continuous function that is caloric everywhere where it is positive, and hence which implies subcaloric.

By homogeneity it is enough to show that $v(x,-1)$ is continuous across the free boundary. Let

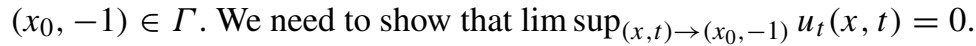

Let $\lim _{j \rightarrow \infty} u_{t}\left(x^{j}+h_{j} y^{j}, t_{j}+h_{j}^{2} s_{j}\right)=\lim \sup _{(x, t) \rightarrow\left(x_{0},-1\right)} u_{t}(x, t)$, where $\left(x^{j}, t^{j}\right) \in \Gamma$ is the closest free boundary point to $\left(x^{j}+h_{j} y^{j}, t_{j}+h_{j}^{2} s_{j}\right)$.

We make the blow-up

$$
u^{j}(x, t)=\frac{u\left(x^{j}+h_{j} x, t_{j}+h_{j}^{2} t\right)}{h_{j}^{2}} .
$$

We may assume that $u^{j} \rightarrow u^{0}$ : if not we can change to a subsequence. Moreover, we have

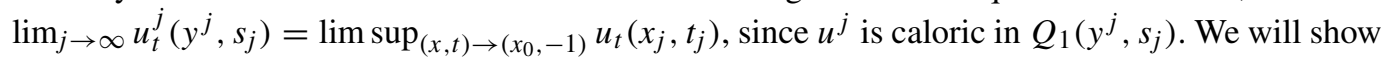
that, for any $\lambda, u^{0}(x, 0)=u^{0}(x, \lambda)$, which in particular implies that $u_{t}^{0}=0$. 
First notice that

$$
u^{0}(x, \lambda)=\lim _{j \rightarrow \infty} \frac{u\left(x^{j}+h_{j} x, t_{j}+h_{j}^{2} \lambda\right)}{h_{j}^{2}} .
$$

By assumption $u\left(r x, r^{2} t\right)=r^{2} u(x, t)$; using this with $r=\sqrt{-t_{j}} / \sqrt{\left|t_{j}+h_{j}^{2} \lambda\right|}$ we may deduce that

$$
u^{0}(x, \lambda)=\lim _{j \rightarrow \infty} \frac{t_{j} u\left(\left(\sqrt{-t_{j}} / \sqrt{\left|t_{j}+h_{j}^{2} \lambda\right|}\right)\left(x^{j}+h_{j} x\right), t_{j}\right)}{\left|t_{j}+h_{j}^{2} \lambda\right| h_{j}^{2}} .
$$

Using $\sqrt{\left|t_{j}\right|} / \sqrt{\left|t_{j}+\lambda h_{j}^{2}\right|}-1 \approx \lambda h_{j}^{2}$ together with the fundamental theorem of calculus we find a point

$$
z^{j}=s x^{j}+(1-s)\left(\sqrt{-t_{j}} / \sqrt{\left|t_{j}+h_{j}^{2} \lambda\right|}\right)\left(x^{j}+h_{j} x\right)
$$

for some $0<s<1$ such that

$$
\left|u^{j}(x, \lambda)-u^{j}(x, 0)\right| \leqslant C \lambda x^{j} \cdot \nabla u^{j}\left(z^{j}, t_{j}\right)+O\left(h_{j}\right) .
$$

Now $\left|x^{j}-z^{j}\right| \leqslant C h_{j}$ and $\left|\nabla u\left(x^{j}, t_{j}\right)\right|=0$ (this follows from $\left(x^{j}, t_{j}\right) \in \Gamma$ ); this together with $C^{1,1}$ estimates implies that $\left|\nabla u^{j}\left(z^{j}, t_{j}\right)\right| \leqslant C h_{j}$. So $\sqrt[6]{6}$ implies that

$$
\left|u^{j}(x, \lambda)-u^{j}(x, 0)\right| \leqslant C \lambda\left|x^{j}\right| \cdot h_{j}+O\left(h_{j}\right) \rightarrow 0 .
$$

This finishes the first step of the proof.

Proof of Step 2. In $[\mathrm{E}]$ it is shown that subcaloric functions are locally bounded from above. In particular $\sup _{Q_{1}} v \leqslant C\|v\|_{L^{1}\left(Q_{2}\right)}$. By homogeneity it follows that $\|v\|_{L^{\infty}} \leqslant C\|v\|_{L^{1}\left(Q_{2}\right)}$.

Proof of Step 3. $\quad v$ is subcaloric and $0 \leqslant v \leqslant C$ in $\left\{x_{1}>0\right\}$ and $v=0$ in $\left\{x_{1}<0\right\}$. Thus by the comparison principle, $v \leqslant w^{T}$ where

$$
\begin{array}{ll}
\Delta w^{T}-w_{t}^{T}=0 & \text { in } \mathbb{R}^{n} \times(-T, 0), \\
w^{T}(x,-T)=\|v\|_{L^{\infty}} & \text { when } x_{1}>0, \\
w^{T}(x,-T)=0 & \text { when } x_{1}<0 .
\end{array}
$$

Letting $T \rightarrow \infty$ it is easy to see that $w^{T} \rightarrow\|v\|_{L^{\infty}} / 2$. Thus $\|v\|_{L^{\infty}} \leqslant\|v\|_{L^{\infty}} / 2$, which clearly implies that $v=0$.

Similarly we can show that

$$
\left(\frac{\partial u(x, t)}{\partial t}\right)^{-}=0
$$

So far we have proved that $u_{t}=0$, but that implies that $\Delta u=\chi_{\{u>0\}}$, so $u$ is a solution to the corresponding elliptic problem and the conclusion follows from $[\mathrm{A}]$.

\section{Proof of Theorem 3}

The proof is the most technically difficult in this paper. We start by proving a lemma which will help us to use Theorem 2 . 
LEMma 3 Let $u \in P_{r}(m, f)$, and assume that $\sup _{Q_{s}^{+}}|u| \leqslant C s^{2}$ for all $s<1$ and some constant $C$ independent of $s$, and that $f$ satisfies Condition 1 Then the blow-up $u^{0}=\lim _{r_{j} \rightarrow 0} u_{r_{j}}$ exists, is homogeneous, $u^{0}\left(r x, r^{2} t\right)=r^{2} u^{0}(x, t)$, and unique.

Proof. Let $r_{j} \rightarrow 0$ be a sequence such that

$$
\lim _{j \rightarrow \infty} \frac{u\left(r_{j} x, r_{j}^{2} t\right)}{r_{j}^{2}}=u_{0}(x, t) \quad \text { locally in } Q_{\infty}^{+}
$$

Then for arbitrary $\alpha$ and $\beta$ we have, by Corollary 1

$$
W_{1}\left(\alpha r_{j}, u\right)-W_{1}\left(\beta r_{j}, u\right) \rightarrow 0 \quad \text { as } j \rightarrow \infty .
$$

But this is equivalent to

$$
0=\lim _{j \rightarrow \infty} W_{1 / r_{j}}\left(\alpha, u_{r_{j}}\right)-W_{1 / r_{j}}\left(\beta, u_{r_{j}}\right)=\lim _{j \rightarrow \infty} \int_{\beta}^{\alpha} \frac{\mathrm{d} W_{1 / r_{j}}\left(\theta, u_{r_{j}}\right)}{\mathrm{d} \theta} \mathrm{d} \theta .
$$

It follows that

$$
\begin{aligned}
0 & =\lim _{j \rightarrow \infty} \int_{\beta}^{\alpha} \frac{1}{2 \theta} \int_{-4}^{-1} \int_{B_{1 /\left(\theta r_{j}\right)}^{+}} \frac{\left|u_{\theta r_{j}}^{\prime}\right|^{2}}{-t} G(x,-t) \mathrm{d} x \mathrm{~d} t \mathrm{~d} \theta \\
& =\lim _{j \rightarrow \infty} \int_{\beta}^{\alpha} \frac{1}{2 \theta^{5}} \int_{-4 \theta^{2}}^{\theta^{2}} \int_{B_{1 / r_{j}}^{+}} \frac{\left|u_{r_{j}}^{\prime}\right|^{2}}{-t} G(x,-t) \mathrm{d} x \mathrm{~d} t \mathrm{~d} \theta .
\end{aligned}
$$

This implies that

$$
\frac{1}{2 \theta^{5}} \int_{-4 \theta^{2}}^{\theta^{2}} \int_{\mathbb{R}_{+}^{n}} \frac{\left|u_{0}^{\prime}\right|^{2}}{-t} G(x,-t) \mathrm{d} x \mathrm{~d} t=0
$$

for almost all $\theta \in(\beta, \alpha)$. This is only true if $u_{0}^{\prime}=0$ a.e., which is equivalent to $u_{0}$ being homogeneous: $u_{0}\left(r x, r^{2} t\right)=r^{2} u_{0}(x, t)$. So by Theorem 2 we conclude that $u_{0}=U_{1}$ or $u_{0}=U_{2}$.

To prove the uniqueness of the blow-up we argue by contradiction. Assume that there exist two subsequences $r_{j}, s_{j} \rightarrow 0$ such that $\lim _{j \rightarrow \infty} u_{r_{j}}=U_{1}$ and $\lim _{j \rightarrow \infty} u_{s_{j}}=U_{2}$. In particular $\lim _{j \rightarrow \infty} W_{a}\left(r_{j}, u\right)=W_{a}\left(1, U_{1}\right)$ and $\lim _{j \rightarrow \infty} W_{a}\left(s_{j}, u\right)=W_{a}\left(1, U_{2}\right)$. Also by Corollary 1 the limit $\lim _{r \rightarrow 0} W_{a}(r, u)$ exists and thus $W_{a}\left(1, U_{1}\right)=W_{a}\left(1, U_{2}\right)$.

We claim that this can only happen if $\lambda=1 / 2$, in which case $U_{1}=U_{2}$. In polar coordinates $x_{1}=$ $r \sin (\phi), x_{2}=r \cos (\phi)$ we have $U_{i}=\frac{r^{2}}{2}\left(\sin \left(\phi+\phi_{i}\right)\right)_{+}^{2}$ where $\phi_{i}=\arctan \left((-1)^{i} \sqrt{1 /(2 \lambda)-1}\right) \in$ $[0, \pi]$. Therefore

$$
\begin{aligned}
W_{a}\left(1, U_{i}\right) & =\int_{-4}^{-1} \int_{B_{a}^{+}}\left(\frac{1}{2}\left|\nabla U_{i}\right|^{2}+U_{i}+\frac{U_{i}^{2}}{2 t}\right) G(x, t) \mathrm{d} x \mathrm{~d} t \\
& =\int_{-4}^{-1} \int_{B_{a}^{+}}\left(\frac{1}{2}|\nabla U|^{2}+U+\frac{U^{2}}{2 t}\right) \chi_{\left\{\phi \in\left(0, \phi_{i}\right)\right\}} G(x, t) \mathrm{d} x \mathrm{~d} t
\end{aligned}
$$


where $U(\phi, t)=\frac{r^{2}}{2} \sin ^{2}(\phi)$. But for $\lambda \in[0,1 / 2]$ we have $\phi_{1} \leqslant \phi_{2}$ with inequality only if $\lambda=1 / 2$. Since we integrate only in the region $\phi \in\left(0, \phi_{i}\right)$ and the integrand is strictly positive it follows that $W_{a}\left(1, U_{1}\right) \leqslant W_{a}\left(1, U_{2}\right)$ with equality only if $\lambda=1 / 2$.

It follows that $W_{a}\left(1, U_{1}\right) \neq W_{a}\left(1, U_{2}\right)$ unless $\lambda=1 / 2$ in which case $U_{1}=U_{2}$. The lemma follows.

We will also need another lemma before we start the proof of the main theorem, but first let us recall a definition from [CPS].

DEFINITION 4 The minimal diameter of a set $E$ in $\mathbb{R}^{n}$, denoted $\operatorname{md}(E)$, is the infimum of distances between two parallel hyperplanes such that $E$ is contained in the strip between these planes.

The importance of this definition is due to the following theorem from [CKS].

THEOREM 4 Let $\Delta u-u_{t}=\chi_{\{u \neq 0\}}$ in $Q_{1}$ and $|u| \leqslant M$. Then there exists a modulus of continuity $\mu$ such that if $\operatorname{md}\left(\Lambda_{u}\left(-r_{0}^{2}\right) \cap B_{r_{0}}\right) / r_{0}>\mu\left(r_{0}\right)$ then $\Gamma_{u}$ is a $C^{1, \alpha}$ graph in $Q_{a r_{0}}$ for some fixed constant $a>0$.

Lemma 4 Let $u$ be as in Theorem 3 Then there exists a $\rho_{\kappa, s}$ for each $1>\kappa, s>0$ such that if $\left(x^{0}, t^{0}\right) \in Q_{\rho_{\kappa, s}}^{+} \cap \Gamma_{u} \cap C_{r_{0}}^{\gamma}$ then

$$
\frac{\operatorname{md}\left(\Lambda_{\tilde{u}}\left(-(s r)^{2}\right) \cap B_{s r}\left(x^{0}, t^{0}-(s r)^{2}\right)\right)}{s r}>\kappa,
$$

where $\tilde{u}=u\left(x+x^{0}, t+t^{0}\right)$ and $r=x_{1}^{0}$.

We will prove the first part of Theorem 3 before we prove this lemma.

Proof of the first part of Theorem 3 Assume that this is not true. That is, we assume that there exists a sequence $u_{j} \in P_{1}(M, f)$ such that $\Gamma_{u_{j}} \cap C^{\gamma} \ni\left(x^{j}, t^{j}\right) \rightarrow 0$ and $\left(x^{j}, t^{j}\right) \notin$ $\left\{(x, t)\right.$; $\left.\operatorname{pardist}\left((x, t), \partial \Omega_{U_{2}}\right)<\epsilon|(x, t)|\right\}$. We make the blow-up

$$
u_{j}(x, t)=\frac{u\left(r_{j} x, r_{j}^{2} t\right)}{r_{j}^{2}} \rightarrow u_{0} \quad \text { for a subsequence in } C^{1, \alpha} .
$$

By assumption $u_{0}$ will be the half-space solution $U_{2}$. Also we have $\left(x^{j} / r_{j}, t^{j} / r_{j}^{2}\right) \rightarrow\left(x^{0}, t^{0}\right)$ and $\operatorname{pardist}\left(\left(x^{0}, t^{0}\right), \Gamma_{U_{2}}\right) \geqslant \epsilon$. Since $u^{j}\left(x^{j}, t^{j}\right)=0$ it follows that $\left(x^{0}, t^{0}\right) \notin \operatorname{spt}\left(U_{2}\right)$ and thus $u_{0}=0$ in $Q_{\epsilon}\left(x^{0}, t^{0}\right)$. But this contradicts the non-degeneracy Lemma 2 and $C^{1, \beta}$ convergence, in particular

$$
0=\sup _{Q_{\epsilon}\left(x^{0}, t^{0}\right)}\left|u_{0}\right|=\lim _{j \rightarrow \infty} \sup _{Q_{\epsilon}\left(x^{j} / r_{j}, t^{j} / r_{j}^{2}\right)}\left|u_{j}\right| \geqslant c \epsilon^{2} .
$$

This finishes the proof.

Proof of Lemma 4 Again we argue by contradiction and blow-up. Assume that there exist $0<$ $s, \kappa_{0}<1$, a sequence $u_{j} \in \hat{P}_{1}(M, f)$ and points $\left(x^{j}, t^{j}\right) \in \Gamma_{u_{j}} \cap Q_{1 / j}^{+}(0)$ such that

$$
\frac{\operatorname{md}\left(\Lambda_{u_{j}\left(x+x^{j}, t+t^{j}\right)} \cap B_{s r_{j}}\left(x^{j}, t^{j}-\left(s r_{j}\right)^{2}\right)\right)}{s r_{j}} \leqslant \kappa_{0}<1
$$


for $r_{j}=x_{1}^{j}$. Upon scaling $u_{j}$,

$$
\tilde{u}_{j}=\frac{u_{j}\left(r_{j} x+x^{j}, r_{j}^{2} t+t^{j}\right)}{r_{j}^{2}},
$$

we will have, for a subsequence, $\tilde{u}_{j} \rightarrow u_{0}$. By part 1 of Theorem 3 we may conclude that in $Q_{R}^{\gamma}$, for each $R>0$,

$$
\operatorname{pardist}\left(\Gamma_{\tilde{u}_{j}}, \Gamma_{U_{2}\left(x+x^{j} / r_{j}\right)}\right) \leqslant \sigma_{\gamma}\left(R r_{j}\right) \rightarrow 0 .
$$

Also $\tilde{u}_{j}\left(-1, x_{2}, \ldots, x_{n}, t\right)=f\left(r_{j} x_{2}+x_{2}^{j}, \ldots, r_{j} x_{n}+x_{n}^{j}, r_{j}^{2} t+t_{j}\right) / r_{j}^{2} \rightarrow \lambda\left(x_{2}+x_{2}^{0}\right)_{+}^{2}$ where $x^{0}=\lim _{j \rightarrow \infty} x^{j} / r_{j}$. It follows that $u_{0}=U_{2}\left(x+x^{0}\right)$. Using non-degeneracy as in (7) and inequality (8) we have

$$
\lim _{j \rightarrow \infty} \operatorname{md}\left(\Lambda_{\tilde{u}_{j}}\left(-s^{2}\right) \cap B_{1}\right)=\operatorname{md}\left(\Lambda_{u_{0}}\left(-s^{2}\right) \cap B_{1}\right) \leqslant \kappa_{0}
$$

But also

$$
\operatorname{md}\left(\Lambda_{u_{0}}\left(-s^{2}\right) \cap B_{1}\right)=\operatorname{md}\left(\Lambda_{U_{2}}\left(-s^{2}\right) \cap B_{1}\right)=1,
$$

which contradicts the previous inequality and finishes the proof.

Proof of the second part of Theorem 3 Lemma 4 implies that if $\left(x^{0}, t^{0}\right) \in \Gamma_{u} \cap C_{r_{0}}^{\gamma}$ and $\left(x^{0}, t^{0}\right)$ is close enough to the origin then

$$
\frac{\operatorname{md}\left(\Lambda_{u_{r}}\left(-s^{2}\right) \cap B_{1}\left(0,-s^{2}\right)\right)}{1}>\kappa \quad \text { where } \quad u_{r}=\frac{u\left(r\left(x-x^{0}\right), r^{2}\left(t-t^{0}\right)\right)}{r^{2}} .
$$

Choosing $s=r_{0}$ and $\kappa=2 \mu\left(r_{0}\right)$ we see that $u_{r}$ satisfies the assumptions in Theorem 4 It follows that $\Gamma_{u_{r}}$ is a $C^{1, \alpha}$ manifold in $Q_{a r_{0}}$. Scaling back we conclude that the normal of $\Gamma_{u}$ is well defined close to the origin. We need to show that the oscillations of the normal of $\Gamma_{u}$ are small close to the origin.

It is enough to exclude that there exists $u_{j}$ and $0 \leftarrow\left(x^{j}, t^{j}\right) \in \Gamma_{u_{j}} \cap C^{\gamma}$ such that the angle of the normal of $\Gamma_{u_{j}}$ at $\left(x^{j}, t^{j}\right)$, which we denote by $\eta_{j}\left(x^{j}, t^{j}\right)$, and the normal of the free boundary of $U_{2}$ goes to zero. To do that we blow up by

$$
\tilde{u}_{j}=\frac{u_{j}\left(r_{j} x-x^{j}, r_{j}^{2} t-t^{j}\right)}{r_{j}^{2}}
$$

where $r_{j}=\left|x_{1}^{j}\right|$. Notice that the normal of $\Gamma_{\tilde{u}_{j}}$ at the origin equals $\eta_{j}\left(x^{j}, t^{j}\right)$. It is therefore enough to show that the normal of $\Gamma_{\tilde{u}_{j}}$ at the origin converges to the normal of $\Gamma_{U_{2}}$.

By the quadratic bound on $u_{j}$ there is a subsequence of $\tilde{u}_{j}$ that converges, in $C^{1, \alpha}$, to some $u_{0}$. By our classification of global solutions, $u_{0}=U_{2}$. But by Lemma 4 we have, for $j$ large enough,

$$
\operatorname{md}\left(\Lambda_{\tilde{u}_{j}}\left(-r_{0}^{2}\right), B_{r_{0}}\right)>r_{0} \mu\left(r_{0}\right),
$$

and thus by Theorem 4 the free boundary $\Gamma_{\tilde{u}_{j}}$ is a $C^{1, \alpha}$ graph in $Q_{a}$. Therefore there exist a subsequence of $j$ 's such that $\Gamma_{\tilde{u}_{j}} \rightarrow \Gamma_{U_{2}}$ in $C^{1, \alpha}$. This implies that the normal of $\Gamma_{\tilde{u}_{j}}$ converges to the normal of $\Gamma_{U_{2}}$ and the second part of the theorem follows. 


\section{REFERENCES}

[A] ANDERSSON, J. On the regularity of a free boundary near contact points with a fixed boundary. J. Differential Equations 323 (2007), 285-302. Zbl 1106.35143 MR 2281197

[AS] Andersson, J., \& Shahgholian, H. Global solutions of the obstacle problem in half-spaces, and their impact on local stability. Calc. Var. Partial Differential Equations 23 (2005), 271-279. Zbl 1090.35177 MR 2142064

[AMU] Apushinsmaya, D. E., Matevosyan, N., \& Uraltseva, N. N. The behavior of the free boundary close to a fixed boundary in a parabolic problem. Indiana Univ. Math. J. 58 (2009), 583-604. Zbl 1173.35124 MR 2514381

[ASU1] Apushinskaya, D. E., Shahgholian, H., \& Uraltseva, N. N. Lipschitz property of the free boundary in a parabolic problem with obstacle. St. Petersburg Math. J. 15 (2004), 375-391. Zbl 1072.35201 MR 2052937

[ASU2] Apushinskaya, D. E., Shahgholian, H., \& Uraltseva, N. N. Boundary estimates for solutions of a parabolic free boundary problem. Zap. Nauchn. Sem. S.-Peterburg. Otdel. Mat. Inst. Steklov. (POMI) 271 (2000), 39-55 (in Russian). Zbl 1031.35150| MR 1810607

[B] BLANK, I. Sharp results for the regularity and stability of the free boundary in the obstacle problem. Indiana Univ. Math. J. 50 (2001), 1077-1112. Zbl 1032.35170 MR 1871348

[C] Caffarelli, L. A. The obstacle problem revisited. J. Fourier Anal. Appl. 4 (1998), 383-402. Zbl 0928.49030 MR 1658612

[CKS] CAffarelli, L. A., Karp, L., \& Shahgholian, H. Regularity of a free boundary with application to the Pompeiu problem. Ann. of Math. (2) 151 (2000), 269-292. Zbl 0960.35112 MR 1745013

[CPS] Caffarelli, L., Petrosyan, A., \& Shahgholian, H. Regularity of a free boundary in parabolic potential theory. J. Amer. Math. Soc. 17 (2004), 827-869. Zbl 1054.35142 MR 2083469

[E] Evans, L. C. Partial Differential Equations. Amer. Math. Soc., Providence, RI (1998). Zbl 0902.35002 MR 1625845

[F] Friedman, A. Variational Principles and Free-Boundary Problems. Krieger, Malabar, FL (1988). Zbl 0671.49001 MR 1009785

[Sh] Shahgholian, H. Null quadrature domains and the modified Schwarz potential.

[SU] Shahgholian, H., \& URAltseva, N. Regularity properties of a free boundary near contact points with the fixed boundary. Duke Math. J. 116 (2003), 1-34. Zbl 1050.35157 MR 1950478

[Wa] Watson, N. A. Thermal capacity. Proc. London Math. Soc. (3) 37 (1978), 342-362. Zbl 0395.35034 MR 0507610

[W] WEISS, G. S. A homogeneity improvement approach to the obstacle problem. Invent. Math. 138 (1999), 23-50. Zbl 0940.35102 MR 1714335 